\title{
GRB Spectral Lags in The Source Frame: An Investigation of Fermi-GBM Bursts
}

\section{E. Sonbas ${ }^{1}$}

University of Adiyaman, Department of Physics, 02040, Adiyaman, Turkey.

E-mail: edasonbas@yahoo.com

T. N. Ukwatta ${ }^{2}$, K.S. Dhuga ${ }^{3}$, A. Shenoy ${ }^{3}$, G. A. MacLachlan ${ }^{3}$, P. N. Bhat ${ }^{4}$, C. Dermer $^{5}$, J. Hakkila ${ }^{6}$, N. Gehrels ${ }^{1}$, W.C. Parke ${ }^{3}$, L.C. Maximon ${ }^{3}$

${ }^{1}$ NASA Goddard Space Flight Center, Greenbelt, MD 20771, USA.

${ }^{2}$ Department of Physics and Astronomy, Michigan State University, East Lansing, MI 48824, USA.

${ }^{3}$ Department of Physics, The George Washington University, Washington, D.C. 20052, USA.

${ }^{4}$ University of Alabama Huntsville, Huntsville, $A L$

${ }^{5}$ Space Science Division, Code 7653, Naval Research Laboratory, Washington, DC 20375, USA.

${ }^{6}$ Department of Physics and Astronomy, College of Charleston, SC 29424-0001

The arrival time differences between high and low energy photons are defined as spectral lags. Many authors have reported a positive correlation between spectral lag and luminosity in both observer and source frames for samples of BATSE and Swift GRBs. We have extracted spectral lags of Fermi GBM bursts in eight source-frame energy bands. Our sample contains 37 GRBs with measured redshifts in the range $z=0.49-8.26$. We find that the lag -luminosity and lag $E_{\mathrm{pk}}$ relations follow a trend similar to that established by the Swift data.

Gamma-Ray Bursts 2012 Conference-GRB2012,

May 07-11, 2012

Munich, Germany 


\section{Introduction}

Spectral lag is defined as the difference between arrival times of high-energy and low-energy photons and considered positive when high-energy photons arrive earlier than low-energy ones. There exists an anti-correlation between spectral lag and the peak luminosity of GRBs [7, 2, 4, 9, 1].

In this work, we have extracted spectral lags of Fermi GBM bursts in eight source-frame energy bands. The energy range of these data is considerably larger than those of previous data. Our sample contains 37 GRBs with measured redshifts in the range $0.49-8.26$. The source-frame energy bands were obtained by projecting variable observer-frame bands to the source-frame [10].

\section{Methodology}

Light Curve Extraction: Background-subtracted light curves were created from the High Time-resolution Time- Tagged Event (TTE) data using the brightest detections combined from NaI and BGO detectors. Background subtracted light curves were extracted for various observer-frame energy bands depending on burst redshift. The fixed source-frame energy bands were selected based on the detectable energy ranges of the NaI and BGO detectors of the Fermi - GBM Instrument (see Table 1).

Table 1: The list of selected source frame energy bands for the analysis

\begin{tabular}{llll}
\hline \hline Band & Energy (keV) & Band & Energy (keV) \\
\hline \hline Band 1 & $100-150 ; 200-250$ & Band 5 & $3850-4650 ; 5450-6250$ \\
Band 2 & $350-450 ; 550-650$ & Band 6 & $7850-9450 ; 11050-12650$ \\
Band 3 & $850-1050 ; 1250-1450$ & Band 7 & $15850-19050 ; 22250-25450$ \\
Band 4 & $1850-2250 ; 2650-3050$ & Band 8 & $31850-38250 ; 44650-51050$ \\
\hline
\end{tabular}

Cross Correlation Function: The CCF method, described in [9], was used to calculate spectral lags. In this method, spectral lags are determined by fitting the global maximum in the cross correlation function by a Gaussian function. The CCF between energy channels $\mathrm{x}$ and $\mathrm{y}$ is defined as:

$$
C C F(d, x, y)=\frac{\sum_{i=\max (1,1-d)}^{\min (N, N-d)} x_{i} y_{i+d}}{\sqrt{\sum_{i} x_{i}^{2}} \sum_{i} y_{i}^{2}}
$$

where, time delay is given by $\tau=\mathrm{d} \times$ time bin size.

Using Band function parameters $\alpha, \beta, \mathrm{E}_{p}$, and observed flux in a given energy band, $\mathrm{E}_{\min }$ and $\mathrm{E}_{\max }$, we also calculated luminosities for 34 long and 3 short GRBs. Uncertainties were calculated using Monte Carlo simulations.

\section{Results}

Lag - Luminosity relation: We have obtained positive lag values for 12 long and one short GRBs. The short GRB (GRB 090510A) has a small lag value for bands 1 and 2. This is consistent with what is already known $[8,11]$ i.e., short GRBs tend to have either small or negligible lag values. In the left panel of Fig. 1, spectral lags and isotropic peak luminosities are plotted in a $\log$-log scale. Black circles represent the lags calculated between Fermi/GBM source frame energy band $100-150 ; 200-250 \mathrm{keV}$ and red circles represent the spectral lags between Swift source frame energy bands $100-150 ; 200-250 \mathrm{keV}$ calculated by [10]. The solid line shows the best-fit power law fit. 


$$
\log \left(\frac{\mathrm{L}_{\text {iso }}}{\operatorname{erg~s}^{-1}}\right)=(55.13 \pm 0.13)-(1.4 \pm 0.06) \log \frac{\operatorname{lag} / \mathrm{ms}}{1+z}
$$

Best-fit power-law indices for each band are consistent with source frame results of [10].
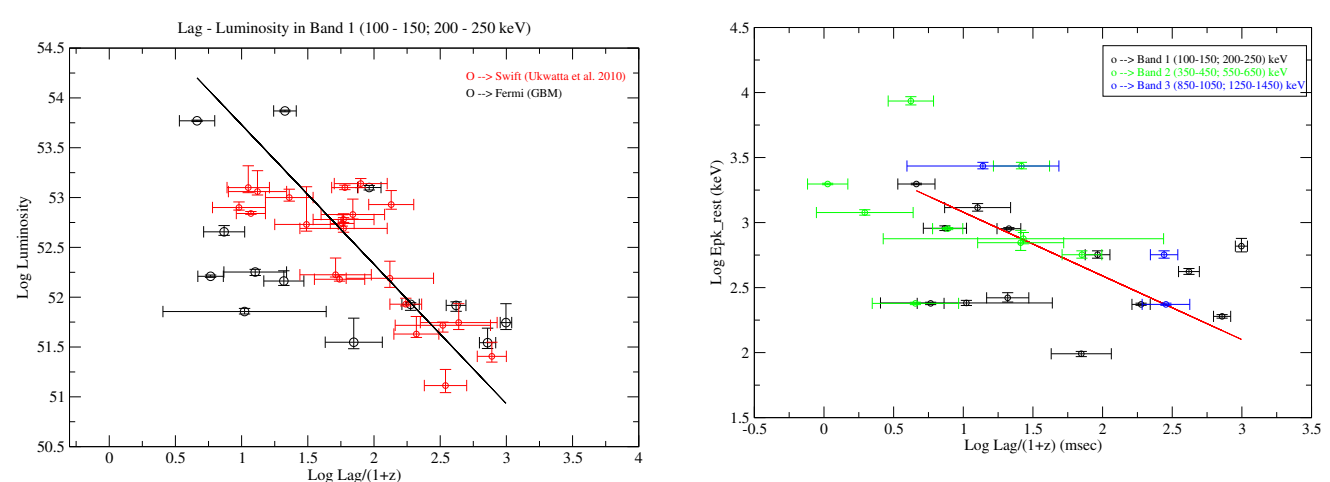

Figure 1: Left Panel: Isotropic peak luminosity vs. spectral lag; solid line represents best fit. Right Panel: Source frame peak energy $\left.E_{\mathrm{pk}}(1+z)\right)$ vs. spectral lag; dashed line represents best fit. Source frame $E_{\mathrm{pk}}$ values were taken from [3].

Lag - $\mathbf{E}_{\mathrm{pk}}$ relation: Spectral lags and source frame peak energy $E_{\mathrm{pk}}$ are plotted in the right panel of Fig. 1. The lags are calculated for Fermi/GBM source frame energy Band 1, Band 2 and Band 3. The solid line represents the best fit. Source frame $E_{\mathrm{pk}}$ values were taken from [3]. The best-fit gives the following relation between $E_{\mathrm{pk}}(1+\mathrm{z})$ and $\mathrm{Lag} /(1+\mathrm{z})$ for Band 1;

$$
\log \left(\frac{E_{\mathrm{pk}}(1+z)}{\mathrm{keV}}\right)=(3.57 \pm 0.05)-(0.49 \pm 0.02) \log \frac{\mathrm{lag} / \mathrm{ms}}{1+z}
$$

The best-fit slope of $-0.49 \pm 0.02$ for Band 1 is consistent with the results of [10].
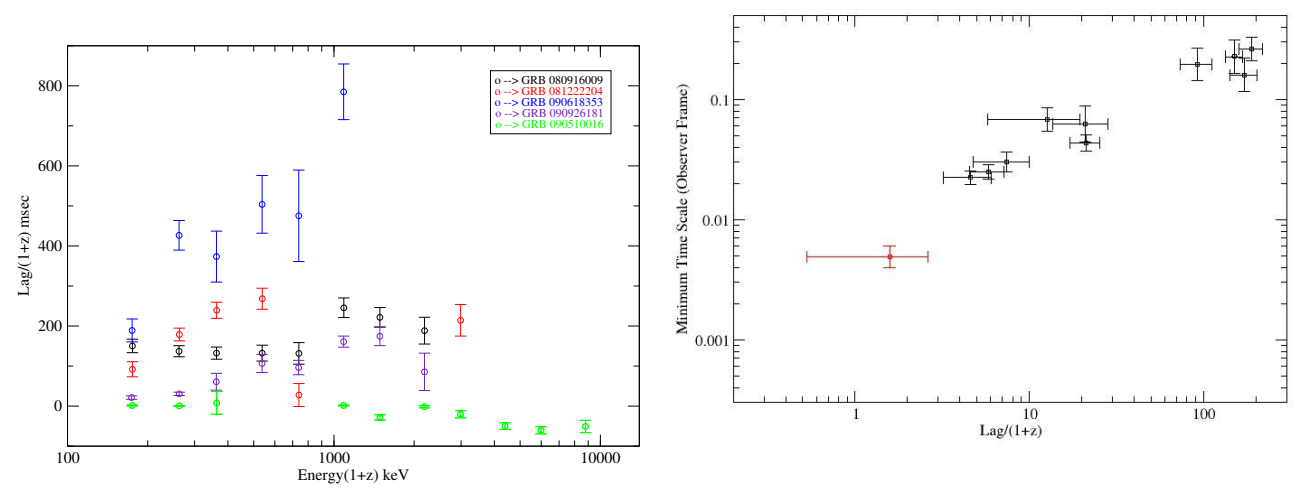

Figure 2: Left Panel: Evolution of spectral lags versus energy for GRB 080916009, GRB 081222204, GRB 090618353, GRB 090926181, and GRB 090510016. Lags calculated respect to the $100-150 \mathrm{keV}$ energy band in the source frame and projected to the observer frame energies. Right Panel: Spectral lags and minimum variability time scales are plotted for band 1 . Red point indicates short GRB in the sample.

Evolution of lag vs energy: We have selected five bright GRBs from the sample in order to see the evolution of lag as a function the source frame energy. The energy bands that we used to extract light curves cover both $\mathrm{NaI}$ and $\mathrm{BGO}$ ranges. We projected them to the observer frame energy bands using the redshift of each burst. Then, we calculated spectral lags of first band with 
respect to the other bands (left panel of Fig. 2). According to prediction of the curvature model by [5], the value of spectral lag is expected to be either constant or zero beyond the value of $E_{\mathrm{pk}}$. While GRB 080916009, GRB 090926181, GRB 0900510016 seem to be consistent with this prediction, GRB 081222204, and GRB 080916353 appear to contradict it.

Lag - variability relation: Employing a fast wavelet technique, [6], obtained a minimum variability timescale (MTS) for a sample of long and short GRBs. Using this timescale, we investigated the lag - variability relation in the source frame for band1 (right panel of Fig. 2). Our results show that there is a positive correlation between MTS and spectral lag.

\section{Conclusions}

We have analyzed the prompt emission for a sample of GBM bursts. The extracted spectral lags, in fixed source-frame energy bands, show features that are consistent with those exhibited by the bursts detected by Swift in the lower energy bands, i.e., the lag-luminosity relation appears to hold. We also notice that $E_{\mathrm{pk}}$ seems to indicate an anti-correlation with the spectral lag.

The evolution of spectral lag in long GRBs as a function of energy is of considerable interest theoretically. Our preliminary results show a number bursts to be consistent with the curvature model, and a number that appear to contradict the model. Further analysis is clearly warranted.

Finally, we exploit the minimum variability timescale extracted by [6] to explore the lagvariability relation. Our results point to a positive correlation between these two temporal features of the prompt emission.

\section{References}

[1] Arimoto, M., et al. Spectral-Lag Relations in GRB Pulses Detected with HETE-2, PASJ 62 (2010) 487

[2] Gehrels, N., et al. A new $\gamma$-ray burst classification scheme from GRB060614, Nature 444 (2006) 1044

[3] Gruber, D., et al. Rest-frame properties of 32 gamma-ray bursts observed by the Fermi Gamma-ray Burst Monitor, A\&A 531 (2011)A20

[4] Hakkila, J.,et al. Correlations between Lag, Luminosity, and Duration in Gamma-Ray Burst Pulses, ApJ 677 (2008) L81

[5] Lu, R.-J., Qin, Y.-P., Zhang, Z.-B., \& Yi, T.-F. Spectral lags caused by the curvature effect of fireballs, MNRAS 367 (2006) 275

[6] MacLachlan, G. et al., Minimum Variability Time Scales of Long and Short GRBs (2012) [arXiv:1201.4431]

[7] Norris, J. P., Marani, G. F., \& Bonnell, J. T. Connection between Energy-dependent Lags and Peak Luminosity in Gamma-Ray Bursts, ApJ 534 (2000) 248

[8] Norris, J. P., \& Bonnell, J. T. Short Gamma-Ray Bursts with Extended Emission, ApJ 643 (2006) 266

[9] Ukwatta, T. N., et al. Spectral Lags and the Lag-Luminosity Relation: An Investigation with Swift BAT Gamma-ray Bursts, ApJ 711 (2010) 1073

[10] Ukwatta, T. N. et al. The lag-luminosity relation in the GRB source frame: an investigation with Swift BAT bursts, MNRAS 419 (2012) 614

[11] Zhang, Z., Xie, G. Z., Deng, J. G., \& Jin, W. Revisiting the characteristics of the spectral lags in short gamma-ray bursts, MNRAS 373 (2006) 729 